\title{
HEREDITARY SEMI-PRIMARY RINGS AND TRI-ANGULAR MATRIX RINGS
}

\author{
MANABU HARADA
}

To the memory of Professor Tadasi Nakayama

It is well known that the semi-simple rings with minimum conditions coincide with the rings of global homological dimension zero and that the hereditary rings coincide with the rings of global dimension one. Eilenberg, Jans, Nagao and Nakayama gave some properties of hereditary rings in [4] and [11], which relate to global dimension of factor rings ${ }^{0 \prime}$. As an example of non-commutative hereditary ring we know a tri-angular matrix ring over a semi-simple ring.

Let $A$ be a ring with radical $N$. If $N$ is nilpotent and $A / N$ satisfies the minimum conditions, then we call $A$ a semi-primary ring.

The purpose of this paper is to give a visible form of hereditary semiprimary rings which is similar to the fact that a simple ring with minimum condition is isomorphic to a matrix ring over a division ring.

In $\S 2$ we shall define a generalized tri-angular matrix ring over semi-simple rings and give properties of such a ring, which is a generalization of [4], Theorem 8 and [11], Proposition 7.

In $\S 3$ we shall show that every hereditary semi-primary ring is isomorphic to a generalized tri-angular matrix ring over semi-simple rings and we show, conversely, every generalized tri-angular matrix ring is a homomorphic image of an hereditary semi-primary ring by modifying slightly the method in [11], $\S 2$.

As an application of results in $\S \S 1-3$, we show in $\S 4$ that if $\Lambda$ is an hereditary semi-primary ring, then so is $e . \lambda e$ for any idempotent $e$ and $A$ con-

Received Dec. 5, 1964.

0) Added in Proof.

Chase generalized those results to a generalized triangular matrix ring in [2] and Nakano also studied such a ring in [4]. Some results in this paper will overlap with them. 
tains a minimal faithful left ideal which is contained in any faithful $A$-projective module as a direct summand.

We consider, in $\S 5$, an hereditary ring with minimum conditions, which is semi-primary. However, we note that we have two ways in such a ring to obtain a relation between hereditary rings and generalized tri-angular matrix rings; namely by using the nilpotency of the radical and the length of composition series of indecomposable left ideals. In general there are no relations between them, however we show that two ways coincide under some assumptions.

We always assume that a ring $\Lambda$ has the unit element and any $\Lambda$-module is unitary. Furthermore, any ring is semi-primary except in $\S 1$.

\section{1. basic rings}

Let $A$ be a ring with unit element 1 and $N$ is the radical of $A . \quad$ In this section we always assume that every idempotent element in $A / N$ is lifted from those elements in $A$, e.g. $A$ satisfies the minimum conditions or $N$ is nil and so on. Furthermore, we assume that $\Lambda / N$ is a semi-simple ring with minimum conditions. In such a situation we have

$$
\Lambda=\sum_{i=1}^{n} \sum_{j=1}^{n(i)} \Lambda e_{i, j}
$$

where the $\Lambda e_{i, j}$ 's are indecomposable left ideals in $\Lambda, e_{i, j}^{2}=e_{i, j}$ and $\Lambda e_{i, j} \approx \Lambda e_{i, k}$, $\Lambda e_{i, j} \neq \Lambda e_{i^{\prime}, k}$ if $i \neq i^{\prime}$. We put $e_{i}=e_{i, 1}$ and $e=\sum_{i} e_{i}$. Then $\tau_{\Lambda}(\Lambda e)=\Lambda$, (see the definition of trace ideal in [1]). Furthermore, it is clear that $\operatorname{Hom}_{\Lambda}^{l}(\Lambda e, \Lambda e)$ $=e \Lambda e$. Since $\tau_{\Lambda}(\Lambda e)=\Lambda$, we obtain from [1], Theorem A. 2 .

Lemma 1. Ae is a finitely generated eAe-projective module and $\Lambda=$ $\operatorname{Hom}_{e \Lambda e}^{r}(\Lambda e, \Lambda e)$.

Proposition 1. Let e be as above. $\Lambda$ is a semi-primary ring such that $N^{t}$ $=(0)$ if and only if so is $e$ e and $N^{\prime t}=(0)$, where $N^{\prime}$ is the radical of $e$ ie.

Proof. Since $N^{\prime}=e N e$, e.le satisfies the above condition if so does $A$. We assume that $\Gamma=e \Lambda e$ is a semi-primary ring with $N^{\prime t}=(0)$. From an exact sequence $0 \rightarrow \Lambda e N^{\prime} \rightarrow \Lambda e \rightarrow \Lambda e / \Lambda e N^{\prime} \rightarrow 0$ we obtain the exact sequence $0 \rightarrow \operatorname{Hom}_{\Gamma}\left(\Lambda e, \Lambda e N^{\prime}\right)$ $\rightarrow \operatorname{Hom}_{\Gamma}(\Lambda e, \Lambda e) \rightarrow \operatorname{Hom}_{\Gamma}\left(\Lambda e, \Lambda e / \Lambda e N^{\prime}\right)=\operatorname{Hom}_{\Gamma / N^{\prime}}\left(\Lambda e / \Lambda e N^{\prime}, \Lambda e / \Lambda e N^{\prime}\right) \rightarrow 0$, since $\Lambda e$ is $\Gamma$-projective. $\Lambda e / \Lambda e N^{\prime}$ is a finitely generated $\Gamma / N^{\prime}$-module by Lemma 1 and hence, $\operatorname{Hom}_{\Gamma / N^{\prime}}\left(\Lambda e / \Lambda e N^{\prime}, \Lambda e / \Lambda e N^{\prime}\right)$ is a semi-simple ring with minimum 
conditions. Therefore, $\operatorname{Hom}_{\Gamma}\left(\Lambda e, \Lambda e N^{\prime}\right)$ contains the radical $N$ of $\Lambda=$ $\operatorname{Hom}_{\Gamma}(\Lambda e, \Lambda e)$. However $\left(\operatorname{Hom}_{\Gamma}\left(\Lambda e, \Lambda e N^{\prime}\right)\right)^{t} \subseteq \operatorname{Hom}_{\Gamma}\left(\Lambda e, \Lambda e N^{\prime t}\right)=(0)$. Hence $A$ is a semi-primary ring such that $N^{t}=(0)$.

Proposition 2. Let $e$ be as above. Then

$$
\begin{aligned}
& \text { 1.gl.dim } \Lambda=1 . g l \cdot d i m e \Lambda e, \\
& \text { r.gl.dim } \Lambda=\text { r.gl.dim } e \Lambda e .
\end{aligned}
$$

Proof. Since $\Lambda e$ is right $e \Lambda e$-projective, we obtain 1 .gl.dim $\Lambda \geq 1$.gl.dim $e \Lambda e$ by [5], Theorem 7. On the other hand 1.gl.dim $e A e \geq 1 . g 1 . \operatorname{dim} \Lambda$ by [7], Lemma 1.2. Replacing $A e$ by $e A$, we have the second half.

Corollary 1. 1 is left hereditary if and only if so is ele. Furthermore, ene/ $N^{\prime}$ is a directsum of division rings.

Remark 1. In Proposition 2 we only need that $\tau_{\Lambda}(\Lambda e)=\Lambda$.

Remark 2. If we consider an hereditary semi-primary ring, we may restrict ourselves to the case where the factor ring with respect to its radical is a direct sum of division rings from the above results.

\section{Generalized tri-angular matrix rings}

From now on we always consider a semi-primary ring $A$ and denote its radical by $N(\Lambda)$. In the next section we shall study an hereditary semi-primary ring and show that it is isomorphic to a generalized tri-angular matrix ring over semi-simple rings (see the below). Thus, we study, in this section, some properties of such a ring.

Let $R_{1}, R_{2}, \ldots, R_{n}$ be rings and $M_{i, j}$ a left $R_{i-}$ and right $R_{j}$-module for $i>j$ and $M_{i, i}=R_{i}$. We consider a family of bilinear $R_{i}-R_{j}$ homomorphisms.

$$
\begin{aligned}
\varphi_{i, j}^{l}: M_{i, l} \otimes_{R_{l}} M_{l, k} \rightarrow M_{i, k} \\
\varphi_{i, t}^{t}: M_{i, t} \otimes_{R_{t}} \approx R_{t} \approx M_{i, t} \\
\varphi_{i, t}^{i}: R_{i}{\underset{R}{R_{i}}}_{M_{i, t}} \approx M_{i, t}
\end{aligned}
$$

and a family of diagrams

$$
\begin{gathered}
M_{i, j} \otimes \mathbb{R}_{R_{j}} M_{j, l} \otimes M_{R l} \stackrel{I_{i, j} \otimes \varphi_{j, k}^{l}}{\longrightarrow} M_{i, j} \otimes M_{R_{j}} M_{j, k} \\
\downarrow \varphi_{i, l}^{j} \otimes I_{l, k} \\
M_{i, l} \otimes_{R l} M_{l, k} \stackrel{\varphi_{i, k}^{l}}{\longrightarrow} M_{i, k}
\end{gathered}
$$


where $I$ means the identity mapping.

Next, we consider the following sets

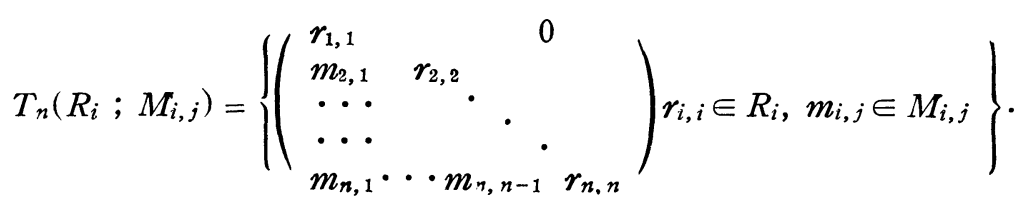

We can make it a ring as follows:

$$
\begin{aligned}
& \left(m_{i, j}\right) \pm\left(m_{i, j}^{\prime}\right)=\left(m_{i, j} \pm m_{i, j}^{\prime}\right) \\
& \left(m_{i, j}\right) \cdot\left(m_{i, j}^{\prime}\right)=\left(\sum \varphi_{i, j}^{l}\left(m_{i, l} \otimes m_{l, j}^{\prime}\right)\right) .
\end{aligned}
$$

It is clear that this product is associative if and only if the diagrams of (2) are commutative.

In this case, we call it a generalized tri-angular matrix ring over $R_{i}$, and we denote it briefly by g.t.a. matrix ring over $R_{i} . \quad M_{i, k} M_{k, j}$ means the image of $M_{i, k} \otimes M_{k, j}$ by $\varphi_{i, j}^{k}$.

We are only interested, in this paper, in a case where all $R_{i}$ are semiprimary. Then a g.t.a. matrix ring over $R_{i}$ is also semi-primary.

LemMa 2. Let $A$ be a g.t.a. matrix ring $T_{n}\left(R_{i} ; M_{i, j}\right)$. If $\Lambda$ is hereditary, then every $R_{i}$-submodule in $M_{i, j}$ is $R_{i}$-projective, and hence, all $R_{i}$ are hereditary.

Proof. Let $M_{i, j}^{\prime}$ be an $R_{i}$-submodule in $M_{i, j}$, or a left ideal in $R$.

$$
\text { Let } L_{0}=\left\{\left(\begin{array}{ll}
0 & 0 \\
\cdot & m_{i, j} \\
\cdot & \cdot \\
0 & 0
\end{array}\right), m_{i, j} \in M_{i, j}^{\prime}\right\} \text {, }
$$

$$
L=\Lambda L_{0} \text { and } \mathfrak{U}=\left(\begin{array}{ccc}
0 & & \\
M_{i, 1} \cdots M_{i, i-1} & 0 & \\
M_{i+1,1} \cdots \cdots & R_{i+1} & 0 \\
\cdots \cdots & & \\
M_{n, 1} \cdots & & R_{n}
\end{array}\right) .
$$

Then $\mathfrak{A}$ is a two-sided ideal in $A$. Since $L$ is $A$-projective, $L / \mathfrak{A} L$ is $A / \mathfrak{A}$-projective. From types of $L / \mathfrak{U} L$ and $\Lambda / \mathfrak{A}$, we know that $M_{i, j}^{\prime}$ is $R_{i}$-projective. Especially we obtain that every left ideal in $R_{i}$ is $R_{i}$-projective. Hence, $R_{i}$ is hereditary.

By replacing $A$ and $L$ by 


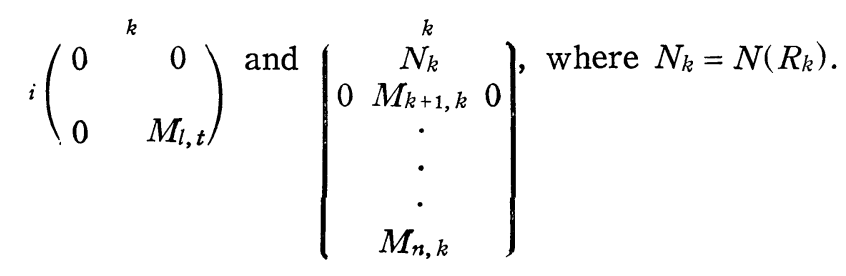

We obtain

Lemma 3. Let $\Lambda$ be as above. If $A$ is hereditary, then $\sum_{t=1}^{i-k+1} M_{i, k+t} M_{k+t, k}$ is a direct summand of $M_{i, k}$ as an $R_{i}$-module for all $>k$, where $M_{i, i}=N\left(R_{i}\right)$.

Lemma 4. Let $\Lambda=T_{n}\left(R_{i} ; M_{i, j}\right)$ and $e_{j}=T_{n}\left(0, \ldots, 0, \hat{1}_{j}, 0, \ldots ; 0\right)$, $e=\sum_{j=i}^{n} e_{j} . \quad$ Let $A$ be a left $\Lambda$-module such that $e A=A . \quad$ Then $1 \cdot \operatorname{dim}_{\Lambda} A=1 \cdot \operatorname{dim}_{e_{\Lambda} e} A$. and gl.dim $\Lambda \geq \operatorname{gl.dim} e \Lambda e$, where $1_{i}$ is the unit element of $R_{i}$.

Proof. It is clear that $\Lambda e=e \Lambda e$. Hence $\Lambda e \underset{e \Lambda e}{\otimes} A=e \Lambda e \underset{e \Lambda e}{\otimes} A=A$ as a left $\Lambda$ module. Since r.dim $\operatorname{din}_{e} \Lambda e=\mathrm{r} \cdot \operatorname{dim}_{e_{\Lambda} e} e \Lambda e=0$, we have $\operatorname{ldim}_{e \Lambda e} A=1 \cdot \operatorname{dim}_{\Lambda} \Lambda e \otimes A$ $=1 \cdot \operatorname{dim}_{\Lambda} A$ and $\operatorname{gl} \cdot \operatorname{dim} \Lambda \geq \operatorname{gl} \cdot \operatorname{dim} e \Lambda e$ by [5], Proposition 15 and Theorem 7 .

THEOREM 1. Let $R_{i}$ be semi-primary rings with radical $N_{i}$. A g.t.a. matrix ring $T_{n}\left(R_{i} ; M_{i, j}\right)$ over $R_{i}$ is hereditary if and only if the following conditions are satisfied.

a) All $R_{i}$ are hereditary,

b) $\varphi_{i, k}^{j}$ is monomorphic,

c) $M_{i, j} / \sum_{t=j}^{i-1} M_{i, t} M_{t, j}\left(\approx \bar{M}_{i, j}\right)$ is $R_{i}$-projective; $\bar{M}_{i, j} \subseteq M_{i, j}$,

d) $M_{i, j}=\bar{M}_{i, j} \oplus M_{i, j+1} \bar{M}_{j+1, j} \oplus \cdots \oplus M_{i, i-1} \bar{M}_{i-1, j} \oplus M_{i, j} N_{j}$ as a left $R_{i}$ module, where $M_{i, i}=N_{i}$.

Proof. Let $\Lambda=T_{n}\left(R_{i} ; M_{i, j}\right)$. It is clear that $N^{\prime}=N(\Lambda)=T_{n}\left(N_{1}, \ldots, N_{n}\right.$; $\left.M_{i, j}\right)$ and $N^{\prime}=\sum \oplus\left(\begin{array}{c}i \\ 0 \\ 0 \\ M_{i} \\ M_{i+1, i} \\ \cdot \\ \cdot \\ \cdot \\ M_{n, i}\end{array}\right)$. We put $L_{i}=\left(\begin{array}{c}N_{i} \\ M_{i+1, i} \\ \cdot \\ \cdot \\ \cdot \\ M_{n, i}\end{array}\right)$

and $\Lambda_{i}=T_{n-i+1}\left(R_{i}, \ldots, R_{n} ; M_{i, j}\right)$. Then we may regard that $L_{i}$ is a left $\Lambda_{i}$ module. Let $e_{i}$ be as above. Then $e_{i} L_{i}=L_{i}$ and $\Lambda_{i}=e_{i} \Lambda e_{i}$. Hence $1 \cdot \operatorname{dim}_{\Lambda} L_{i}$ $=1 \cdot \operatorname{dim}_{\Lambda_{i}} L_{i}$ by Lemma 4 . Therefore, we know that $A$ is hereditary if and only 
if $L_{i}$ is $A_{i}$-projective for all $i$. For the sake of simpleness to explain, we consider a case of $i=1$. We denote $L_{1}, \Lambda_{1}$ by $L, \Lambda$. Then

$$
L=L / N L=\left(\begin{array}{c}
M_{1,1}^{*} \\
M_{2,1}^{*} \\
\cdot \\
\cdot \\
\cdot \\
M_{n, 1}^{*}
\end{array}\right)
$$

where $M_{1,1}^{*}=N_{1} / N_{1}^{2}, M_{i, 1}^{*}=M_{i, 1} /\left(M_{i, 1} N_{1}+\cdots+N_{i} M_{i, 1}\right)$.

Let $\left\{f_{k}^{(j)}\right\}$ be a set of non-isomorphic primitive idempotent elements in $R_{j}$. We put $\tilde{f}_{k}^{(j)}=T_{n}\left(0, \ldots, 0, f_{k}^{(j)}, \ldots, 0 ; 0\right)$. Since $M_{i, 1}^{*}$ is an $\bar{R}_{i}=R_{i} / N_{i}$ module, $M_{i, 1}^{*}=\sum_{t} \oplus L_{t}^{(i)} ; L_{t}^{(i)}=\bar{R}_{i} y_{t}^{(i)} \approx \bar{R}_{i} f_{l(t)}^{(i)}, y_{t}^{(i)} \in M_{i, 1}$. From the idea of minimal projective resolution (cf. [3]), we know that $L$ is $\Lambda$-projective if and only if

$$
\begin{gathered}
L=\sum_{i} \sum_{t} \oplus \Lambda \tilde{y}_{t}^{(i)}, \text { where } \tilde{y}_{t}^{(i)}=\left(\begin{array}{r}
0 \\
\cdot \\
\cdot \\
0 \\
y_{t}^{(i)} \\
0 \\
\cdot \\
\cdot
\end{array}\right) \text { and } \\
\Lambda \tilde{y}_{t}^{(i)}=\left(\begin{array}{l}
0 \\
0 \\
R_{i} y_{t}^{(i)} \\
M_{i+1, i} y_{t}^{(i)} \\
M_{n, i} y_{t}^{(i)}
\end{array}\right) \approx \Lambda \tilde{f}_{l(t)}^{(i)}=\left(\begin{array}{c}
0 \\
0 \\
R_{i} f_{l(t)}^{(i)} \\
M_{i+1, i}^{(i)} \\
M_{n, i}^{(i)} f_{l(t)}^{(i)}
\end{array}\right)
\end{gathered}
$$

(natural isomorphism).

Let $M_{i, 1}^{* *}=\sum_{t} R_{i} y_{t}^{(i)} \subseteq M_{i, 1}$. We assume that $A$ is hereditary. Then we obtain $M_{i, 1}=P_{i} \oplus\left(M_{i, 1} N_{1}+\cdots+M_{i, i-1} M_{i-1,1}\right)$ for some $R_{i}$-module $P_{i}$ from Lemma 3. Hence $M_{i, 1} N_{1}+\cdots+M_{i, i-1} M_{i-1,1}+N_{i} M_{i, 1}=N_{i} P_{i} \oplus\left(M_{i, 1} N_{1}+\cdots+M_{i, i-1} M_{i-1,1}\right)$. Therefore, we may assume that $y_{t}^{(i)} \in P_{i}$. Then $P_{i}=M_{i, 1}^{* *}=M_{i, 1}$ since $N_{i}$ is nilpotent. Now, 4) is equivalent to facts that $\bar{M}_{i, 1}=\sum_{t} \oplus R_{i} y_{t}^{(i)}$ and that $\sum_{t} M_{l, i} y_{t}^{(i)}$ and $\sum_{i=1}^{l-1} M_{l, i} \bar{M}_{i, 1}+\bar{M}_{l, 1}$ are direct sums, respectively. The above arguments are true by replacing 1 by any $h$. Furthermore, we obtain from (2)

$$
M_{k, i} M_{i, j} M_{i, h} \subseteq M_{k, j} M_{j, h} \text { for all } k \geq i \geq j \geq h .
$$


Since $M_{k, i} \otimes M_{i, j}=M_{k, i} \otimes \bar{M}_{i, j} \oplus M_{k, i} \otimes\left(\sum_{t=j}^{i-1} M_{i, t} M_{t, j}\right)$, we obtain from (6)

$$
\begin{aligned}
M_{k, i} M_{i, j} \subseteq M_{k, i} \bar{M}_{i, j}+\sum_{t=j}^{i-1} M_{k, t} M_{t, j} \subseteq M_{k, i} \bar{M}_{i, j}+ \\
M_{k, i-1} \bar{M}_{i-1, j}+\sum_{t=j}^{i-2} M_{k, t} M_{t, j} \subseteq \sum_{s=j+1}^{i} M_{k, s} M_{s, j}+M_{k, j} N_{j} .
\end{aligned}
$$

Therefore,

$$
M_{k, j} N_{j}+M_{k, j+1} M_{j+1, j}+\cdots+M_{k, k-1} M_{k-1, j} \subseteq \sum_{s=j+1}^{k-1} M_{k, s} \bar{M}_{s, j}+M_{k, j} N_{j} .
$$

Hence $M_{k, j}=\sum \oplus M_{k, s} \bar{M}_{s, j} \oplus M_{k, j} N_{j} \oplus \bar{M}_{k, j}$ from the above observation. Which shows d), a) and c) by Lemmas 2 and 3. Furthermore, from (5) we obtain $f_{l(t)}^{(1)} y_{t}^{(1)}=y_{t}^{(1)}$. Hence (4) and (5) implies $\varphi_{i, 1}^{j}$ is monomorphic on $M_{i, j} \otimes \bar{M}_{j, 1}$. Using this argument we shall show that $\varphi_{i, k}^{j}$ is monomorphic for all $i \geq j \geq k$. Let $\Gamma=T_{n-j+1}\left(R_{j}, \ldots, R_{n} ; M_{l, s}\right)$ and $L=\left(\begin{array}{c}0 \\ M_{j, k} \\ M_{n, k}\end{array}\right)$. Since $\Gamma$ is hereditary by lemma 4, we know from the above argument that $\varphi_{i, k}^{j}$ is monomorphic on $M_{i, j} \otimes \bar{M}_{j, k}$. However, $\bar{M}_{j, k}=M_{j, k}$ in this case, and hence, $\varphi_{i, k}^{j}$ is monomorphic on $M_{i, j} \otimes M_{j, k}$.

Conversely we assume that a)-d) are satisfied. From a) we have $\sum_{t} \oplus R_{1} y_{t}^{(1)}$ $=N_{1}$. From a), c) and the remark after (5), we obtain $M_{i, 1}^{* *}=\bar{M}_{i, 1}=\sum_{i} \oplus R_{i} y_{t}^{(i)}$. Since $\varphi_{l, 1}^{i}$ is monomorphic on $M_{l, i} \otimes \bar{M}_{i, 1}$ by b), we have (5) from d). Furthermore, d) and a) imply (4).

Corollary 2. Let $\Lambda$ be a g.t.a. matrix ring over semi-simple rings. If $\varphi_{i, k}^{j}$ is isomorphic, then $A$ is hereditary.

Proof. From the assumption, we have $\bar{M}_{i, i-1}=M_{i, i-1}$ for all $i$ and $\bar{M}_{i, j}=$ (0) for all $i>j+1$. Hence $\Lambda$ satisfies a)-d).

Remark 3. An usual tri-angular matrix ring over a semi-simple ring $R$ is a special case of Corollary 2 .

Theorem 2. Let $R_{i}$ be semi-primary rings and $M_{i, j} R_{i}-R_{j}$ modules. Let $\Lambda_{i}=T_{n-i+1}\left(R_{i}, \ldots, R_{n} ; M_{l, j}\right)$. Then gl.dim $\Lambda_{i+1} \leq \operatorname{gl} . \operatorname{dim} \Lambda_{i} \leq \operatorname{gl} . \operatorname{dim} \Lambda_{i+1}+$ gl.dim $R_{i}+1$.

Proof. From Lemma 4, we obtain gl.dim $\Lambda_{i+1} \leq$ gl.dim $\Lambda_{i}$. We denote $\Lambda_{i}$, $\Lambda_{i+1}$ by $\Lambda, \Gamma$. Then $N=N(\Lambda)=T_{n-i+1}\left(N_{i}, \ldots, N_{n} ; M_{i, j}\right)$, where $N_{i}=N\left(R_{i}\right)$. 
$N=L_{i} \oplus N(\Gamma)$ as a left $\Lambda$-module, where $L_{i}$ is the same as in the proof of Theorem 1. From Lemma 4 we have gl.dim $\Lambda=1+1 \cdot \operatorname{dim}_{\Lambda} N=1+\sup \left(1 \cdot \operatorname{dim}_{\Lambda}\right.$ $L_{i}, \operatorname{dim}_{\Gamma} N(\Gamma)$ ). If we use the same notations as in the proof of Theorem 1 , then

$$
0 \rightarrow \varphi^{-1}(0) \rightarrow \sum_{j=i}^{n} \sum_{s} \Lambda \tilde{f}_{s}^{(j)}=\sum \oplus P_{0}^{j} \rightarrow L_{i} \rightarrow 0
$$

is exact, where $P_{0}^{j}=\sum_{s} \Lambda f_{s}^{(j)}$. We consider $P^{(i)}=\sum_{s} \oplus \Lambda f_{s}^{(i)} \stackrel{\varphi(i)}{\rightarrow} 1\left(\begin{array}{c}N_{i} \\ 0 \\ \cdot \\ \cdot \\ 0\end{array}\right) \rightarrow 0$. Then $\varphi^{(i)-1}(0)=\left(\begin{array}{l}K_{i} \\ N_{i+1, i}^{\prime}\end{array}\right)$, where $0 \rightarrow K_{i} \rightarrow \sum_{s} \oplus R_{i} f_{s}^{(i)} \rightarrow N_{i} \rightarrow 0$ is exact and $N_{j, i}^{\prime}$ is an $\left|\begin{array}{c}N_{i+1, i}^{\prime} \\ \cdot \\ \cdot \\ N_{n, i}^{\prime}\end{array}\right|$

$R_{j}$-module. Hence we can see directly that $\varphi^{-1}(0)=\left(\begin{array}{l}K_{i} \\ N_{i, 1, i}\end{array}\right) ; N_{j, i}$ is an

$$
\left(\begin{array}{c}
K_{i} \\
N_{i+1, i} \\
\cdot \\
\cdot \\
N_{n, i}
\end{array}\right)
$$

$R_{j}$-module. If we repeat this argument on $K_{i}$ we have a minimal $\Lambda$-projective resolution of $L_{i}$ :

$$
\longrightarrow \sum \oplus P_{k}^{j} \stackrel{d_{k}}{\longrightarrow} \cdots \longrightarrow \sum \oplus P_{1}^{j} \stackrel{d_{1}}{\longrightarrow} \sum \oplus P_{0}^{j} \stackrel{d_{0}}{\longrightarrow} L_{i} \longrightarrow 0
$$

and the first row of each $P^{i}$ forms a minimal $R_{i}$-projective resolution of $N_{i}$. Hence if gl.dim $R_{i}=m$, then $d_{m-1}^{-1}(0)$ is a $\Gamma$-module. Hence $1 \cdot \operatorname{dim}_{\Lambda} d_{m}^{-1}(0) \leq \mathrm{gl}$.dim $\Gamma$ by Lemma 4 . Therefore, $1 \cdot \operatorname{dim}_{\Lambda} N \leq \operatorname{gl} \cdot \operatorname{dim} R+\operatorname{gl} \cdot \operatorname{dim} \Gamma$. Thus, we obtain gl.dim $\Lambda \leq$ gl.dim $R_{i}+$ gl.dim $\Gamma+1$.

Corollary 3. We assume that all $R_{i}$ are semi-simple rings in the above. Then gl.dim $\Lambda_{i+1} \leq$ gl.dim $\Lambda_{i} \leq 1+$ gl.dim $\Lambda_{i+1}$ and gl.dim $\Lambda \leq n-1$.

Let $\Lambda$ be a g.t.a. matrix ring $T_{n}\left(R_{i} ; M_{i, j}\right)$ and $e_{i}$ be as in Lemma 4 . For an element $a$ in a two-sided ideals $\mathfrak{U}$ in $\Lambda e_{i} a e_{j} \in \mathfrak{U}$. Hence $\mathfrak{U}=T_{n}\left(S_{i} ; N_{i, j}\right)$, where $S_{i}$ is an ideal in $R_{i}$ and $N_{i, j}$ is an $R_{i}-R_{j}$ module in $M_{i, j}$. Hence we obtain from Corollary 3.

THEOREM 3. Let $\Lambda$ be a g.t.a. matrix ring $T_{n}\left(R_{i} ; M_{i, j}\right)$ over semi-simple 
rings $R_{i}$. Then for any two-sided ideal $A$ in $A$ we have

$$
\text { gl.dim } \Lambda / \mathfrak{A} \leq n-1 \text {. }
$$

Furthermore, we assume that all $R_{i}$ are simple rings and $\mathfrak{A}+N / N \approx \sum_{t=1}^{s} \oplus R_{i_{t}}$. Then gl.dim $1 / \mathfrak{A} \leq \boldsymbol{n}-\boldsymbol{s}-1$, where $N$ is the radical of $\Lambda$.

\section{Hereditary semi-primary rings}

In this section we shall determine a type of hereditary semi-primary rings. Let $\Lambda$ be such a ring and $N$ the radical. By virtue of Remark 2, we shall first restrict ourselves to the case where $1 / N$ is a direct sum of division rings. Then we obtain $1=\sum e_{i}$, where $\left\{e_{i}\right\}$ is a set of non-isomorphic primitive idempotent elements in $A$. Since $N^{t}=(0)$ for some $t$, we have an integer $s$ for $e_{i}$ such that $N^{s} e_{i} \neq(0), N^{s+1} e_{i}=(0)$. We denote such an integer $s$ by $n\left(e_{i}\right)$. Then we can rearrange $e_{i}$ as follows: $n\left(e_{i}\right) \geq n\left(e_{i+1}\right)$ for all $i$.

We quote here a well known results by [3] and [12].

Lemma 5. Let $A$ be an hereditary semi-primary ring. Then every A-projective module is isomorphic to $\sum \oplus\left(\Lambda e_{i}\right)^{\left.s_{i} 1\right)}$.

Lemma 6. Let $A$ be as above. If $e$ is an idempotent element in $A$ such that $n(e)$ is minimal among $n\left(e_{i}^{\prime}\right)$, where $e^{\prime}$ runs through all primitive idempotent elements in A. Then $n(e)=0$.

Proof. If $N e \neq(0)$, then $N e=\sum \oplus\left(A \boldsymbol{e}_{i}\right)^{s_{i}}$ by Lemma 5. Hence $n(\boldsymbol{e})>n\left(\boldsymbol{e}_{i}\right)$, which is a contradiction.

Lемма 7. Let $\Lambda$ be an hereditary semi-primary ring such that $\Lambda / N \approx \sum \oplus$ $\Delta_{i} ; \Delta_{i}$ division ring. Then $e_{i} N e_{j}=(0)$ for $i \leq j$ and $e_{i} \Lambda e_{i} \approx \Delta_{i}$.

Proof. From Lemma 6 we know that $N e_{n}=(0)$. Therefore, $e_{i} N e_{n}=(0)$ for all $i$. We assume that $e_{i} N e_{j}=(0)$ for $i \leq j$ and $j>k$. Since $N e_{k}$ is $\Lambda$ projective, we have from Lemma 5 and the assumption $n\left(\boldsymbol{e}_{i}\right) \geq n\left(\boldsymbol{e}_{i+1}\right)$

$$
N e_{k} \approx \sum_{l>k} \oplus\left(A e_{l}\right)^{s_{l}}
$$

Hence, $e_{i} N e_{k} \approx \sum \oplus\left(e_{i} \Lambda e_{l}\right)^{s_{l}}=\sum \oplus\left(e_{i} N e_{l}\right)^{s_{l}}=(0)$ for $\imath \leqq k$ from the above assumption. Therefore, $e_{i} N e_{j}=(0)$ for all $i \leq j$. Since $e_{i} N e_{i}=(0), \Delta_{i} \approx \boldsymbol{e}_{i} / \boldsymbol{e}_{i} N \boldsymbol{e}_{i}$ $=e_{i} 1 e_{i}$.

${ }^{11} M^{n}$ means a directsum of $n$ copies of $M$. 
THEOREM 4 '. Let $A$ be an hereditary semi-primary ring such that $A / N \approx$ $\sum_{i=1}^{n} \oplus \Delta_{i}$. Then $\Lambda$ is isomorphic to a g.t.a. matrix ring over $\Delta_{i}$, where $N$ is the radical of $A$ and the $\Delta_{i}^{\prime}$ 's are division rings, ([2] and [14]).

Proof. Since $1=\sum_{i=1}^{n} e_{i}, \Lambda=\sum_{i, j} \oplus e_{i} \Lambda e_{j}$ and $e_{i} \Lambda e_{j}=(0)$ if $i<j . \quad$ If we put $M_{i, j}=e_{i} \Lambda e_{j}$ and $\varphi_{i, k}^{j}$ is a product of $M_{i, j}$ and $M_{j, k}$, then $\Lambda=T_{n}\left(\Delta_{1}, \ldots, \Delta_{n}\right.$; $M_{i, j}$ ) by Lemma 7 .

We shall generalize the above theorem.

Let $\Lambda=T_{n}\left(R_{i} ; M_{i, j}\right)$ be a g.t.a. matrix ring over rings $R_{i}$. Let

$$
\left.\mathfrak{M}_{i, j}=\left(\begin{array}{c}
\overbrace{M_{i, j} \cdots}^{s_{j}} M_{i, j} \\
\cdots \\
M_{i, j} \cdots M_{i, j}
\end{array}\right)\right\}^{s_{i}} \equiv M_{i, j}\left(s_{i} \times s_{j}\right)
$$

Then we can define a natural operation of elements in $\left(R_{i}\right)_{s_{i}}$ (resp. $\left.\left(R_{j}\right)_{s_{j}}\right)$ from the left side (resp. right side) on $\mathfrak{M}_{i, j}$. We put $\Gamma=T_{n}\left(\left(R_{1}\right)_{s_{1}}, \ldots,\left(R_{n}\right)_{s_{n}}\right.$; $\left.\mathfrak{M}_{i, j}\right)$, then $\Gamma$ is also a g.t.a. matrix rings over $\left(R_{t}\right)_{s_{t}}$ with naturally extended bi-linear mapping $\Phi_{i, k}^{j}:\left(\left(x_{t, p}\right)\right) \underset{(R p) s_{p}}{\otimes}\left(\left(y_{r, q}\right)\right) \rightarrow\left(\left(\sum \varphi_{i, k}^{j}\left(x_{t, p} \otimes y_{p, q}\right)\right), x_{t, p} \in M_{i, j}\right.$, $y_{r, q} \in M_{j, k}$.

Let $e_{l, m}^{(i)}$ be the matrix units in $\left(R_{i}\right)_{s_{i}}$ and $E_{i}=T_{n}\left(0, \ldots, 0 e_{e_{1,1}}^{i}, 0 \cdots 0 ; 0\right)$. If we put $E=\sum E_{i}$ then $\tau_{\Gamma}(\Gamma E)=\Gamma$ and $E I^{\prime} E \approx \Lambda$. Hence we have from Proposition 2

$$
\text { gl.dim } \Lambda=\operatorname{gl} \operatorname{dim} \Gamma \text {. }
$$

We call $\Gamma$ an induced g.i.a. mairix ring from $A$.

ThEOREM $4 "$. Let $A$ be an hereditary semi-primary ring. Then $A$ is isomorphic to an induced g.t.a. matrix ring $T\left(R_{i} ; \mathfrak{M}_{i, j}\right)$ over simple rings $R_{i}$ from a g.t.a. matrix ring as in Theorem $4^{i} . \quad \Phi_{i, k}^{j}$ is monomorphic for all $i \geq j \geq k$ and $\mathfrak{M}_{i, j+1} \overline{\mathfrak{M}}_{j+1, j}+\cdots+\mathfrak{M}_{i, i-1} \overline{\mathfrak{M}}_{i-1, j}$ is a directsum in $\mathfrak{M}_{i, j}$ as a left $R_{i}$-module, where $\mathfrak{M}_{i, j}=\overline{\mathfrak{M}}_{i, j} \oplus \sum_{t=j+1}^{i-1} \mathfrak{M}_{i, t} \mathfrak{M}_{t, j}$ as a left $R_{i}$-module.

Proof. Let $\Lambda / N=\sum \oplus R_{i}$ and $e=\sum e_{i}$ as in $\S 1$. Then $\Lambda=\operatorname{Hom}_{e \Lambda e}(\Lambda e, \Lambda e)$ and $e \Lambda e$ is an hereditary semi-primary ring by Propositions 1 and 2 . Furthermore, $\Lambda e$ is a finitely generated $e \Lambda e$-projective module, and hence,

$$
\Lambda e_{i} \approx \sum \oplus\left(f_{i} \Gamma\right)^{s_{i}}
$$


where $\Gamma=e \Lambda e$, and $\left\{f_{l}\right\}$ is a set of non-isomorphic primitive idempotent elements in $\Gamma$ and all $s_{i}$ are finite. We may assume $n\left(f_{i}\right) \geq n\left(f_{i+1}\right)$ for all $i$. Hence it is clear that $\Lambda=T_{n}\left(\left(f_{1} \Gamma f_{1}\right)_{s_{1}}, \ldots,\left(f_{n} \Gamma f_{n}\right)_{s_{n}} ;\left(f_{i} \Gamma f_{j}\left(s_{i} \times s_{j}\right)\right)\right.$. The second part is clear from Theorem 1.

We shall modify slightly the above theorem.

LEMMA 8. If $n\left(e_{i}\right)=n\left(e_{i+1}\right)=\cdots=n\left(e_{i+s}\right)$, then $e_{k} \Lambda e_{p}=(0)$ for $k \leq i+s$, $i \leq p \leq i+s-1$ and $k \neq p$.

Proof. Since $N e_{p}=\sum \oplus\left(\Lambda e_{l}\right)^{q_{l}}$ for $i \leq p \leq i+s$ from the assumption, $e_{k} N e_{p}$ $=\sum_{l \geq l+s+1} \oplus\left(e_{k} \Lambda e_{l}\right)^{q_{l}}$. Hence if $k \leq i+s, e_{k} \Lambda e_{l}=(0)$ by Lemma 7 .

Lemma 9. $n\left(e_{i+1}\right) \leq n\left(e_{i}\right) \leq n\left(e_{i+1}\right)+1$.

Proof. Let $t=n\left(e_{i+1}\right) . \quad N e_{i}=\sum_{j z_{i}+1} \oplus\left(\Lambda e_{j}\right)^{s_{j}}$. Then $N^{t+2} e_{i}=\sum_{j \geq_{i+1}}\left(N^{t+1} e_{j}\right)^{s_{j}}$ $=(0)$. Hence $n\left(e_{i}\right) \leq t+1$.

First we assume that $\Lambda$ is an hereditary semi-primary ring such that $A / N$ $=\sum \oplus \Delta_{i}$. We assume $N^{n-1} \neq(0), N^{n}=(0)$. Then it is clear that $n\left(e_{1}\right)=n-1$. If we classify $e_{i}^{\prime} s$ by a relation $e \sim e^{\prime} \Longleftrightarrow n(e)=n\left(e^{\prime}\right)$, then we have $(n-1)$ classes by Lemma 9. Furthermore, if $e_{i}, \ldots, e_{i+t-1}$ are in a class then we put $R_{i}=e_{i} \Lambda e_{i} \oplus \cdots \oplus e_{i+t-1} \Lambda e_{i+t-1}$. Then $\Lambda=T_{n}\left(R_{i} ; \mathfrak{M}_{i, j}\right)$, where

$$
\mathfrak{M}_{i, j} \equiv\left(\begin{array}{c}
M_{i, j} M_{i, j+1} \cdots M_{i, j+t-1} \\
M_{i+1, j} \cdots \cdots M_{i+1, j+t-1} \\
\cdots \cdots \cdots \\
M_{i+t-1, j} \cdots \cdots M_{i+t-1, j+t-1}
\end{array}\right)
$$

and $N=\mathfrak{M}_{n, n-1} \mathfrak{M}_{n-1, n-2} \cdots \mathfrak{M}_{2,1} \neq(0)$. Therefore, $\mathfrak{M}_{i, j} \supseteq \mathfrak{M}_{i, i-1} \mathfrak{M}_{i-1, i-2} \cdots \mathfrak{M}_{j+1, j}$ $\neq(0)$. Hence we have in general.

THEOREM $4^{\prime \prime \prime}$. Let $\Lambda$ be an hereditary semi-primary ring such that $N^{n-1} \neq(0)$, $N^{n}=(0)$. Then $A$ is isomorphic to an induced g.t.a. matrix ring $T_{n}\left(R_{1}, \ldots\right.$, $\left.R_{n} ; \mathfrak{M}_{i, j}\right)$ over semi-simple rings such that all $M_{i, j} \neq(0)$. Furthermore, $T_{n-i+1}\left(R_{i}, \ldots, R_{n} ; \mathfrak{M}_{i, j}\right)$ is also an hereditary semi-primary ring with radical $N_{i}$ such that $N_{i}^{n-i} \neq(0), N_{i}^{n-i+1}=(0)$.

Remark 4. The expression of 1 in Theorem $4^{\prime \prime \prime}$ is not unique. For example,

$$
A=\left(\begin{array}{llll}
\Delta & & & \\
0 & \Delta & & 0 \\
\Delta & 0 & \Delta & \\
\Delta & \Delta & \Delta & \Delta
\end{array}\right)
$$


Then $A$ is hereditary by Corollary 2. However we have two expressions as Theorem 4"' :

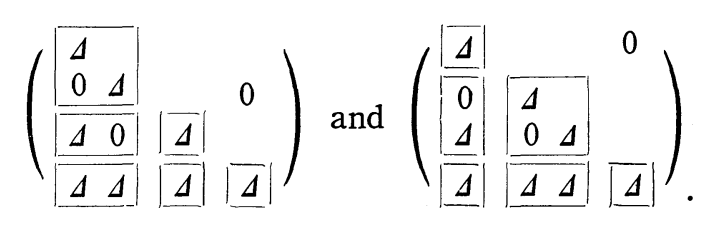

The latter is as in Theorem $4^{\prime \prime \prime}$.

Corollary 4. Let $\Lambda$ be as above. Then for any two-sided ideal $\mathfrak{A}$ in $\Lambda$

$$
\text { gl. } \operatorname{dim} \Lambda / \mathfrak{A} \leq n-1 \text {. }
$$

We shall give one more remark for this expression.

From Theorem 4", we do not lose a generality if we consider a case of $\Lambda / N=\sum \oplus \Delta_{i} . \quad$ If $n\left(e_{i}\right)=n\left(e_{i+1}\right)=\cdots=n\left(e_{i+t}\right)>n\left(e_{i+t-1}\right)=\cdots=n\left(e_{i+t+t}\right)$ $>\cdots$ then for any $j$ such that $i \leq j \leq i+t$ we have $N e_{j}=\Lambda e_{s} \oplus \cdots$, where $i+t+1 \leq s \leq i+t+t^{\prime}$. Hence $e_{s} N e_{j} \neq(0)$. Therefore, if $M_{i, j}$ is as in (8), then

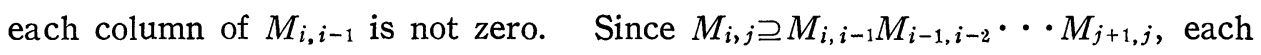
column of $M_{i, j}$ is not zero. Conversely, in an expression in Theorem 4'", if we assume that each column of $M_{i, i-1}$ is not zero, then each unit element $e$ of simple components of $R_{i}$ has the same $n(e)$ and converse. Under such an assumption we have a unique expression up to isomorphism.

We call such a representation of hereditary ring a left normal representation as a g.t.a. matrix ring.

If we start from properties of $e N$ instead of $N e$, we have the similar arguments as above.

By $n^{\prime}(e)$ we denote an integer such that $e N^{n} \neq(0), e N^{n+1}=(0)$. In general, there are no relations between $n(e)$ and $n^{\prime}(e)$. For instance

$$
\Lambda=\left(\begin{array}{llll}
\Delta & & & 0 \\
\Delta & \Delta & & \\
0 & 0 & \Delta & \\
\Delta & \Delta & \Delta & \Delta
\end{array}\right) . \quad \text { Then } n\left(e_{3}\right)=1 \text { and } n^{\prime}\left(e_{3}\right)=0 .
$$

However from the above observation we have

PROPOSITION 3. Let $A$ be an hereditary semi-primary ring such that $N^{n-1} \neq(0)$, $N^{n}=(0)$. Then for any idempotent element $e \quad n(e)=n-n^{\prime}(e)$ if and only if $A$ has a right and left normal representation as a g.t.a. matrix ring. 
Finally we give a characterization of a g.t.a. matrix ring over semi-simple rings. We recall the definition of connected sequence of primitive idempotents, (cf. [11], §1. We do not need an assumption $\left.N^{2}=(0)\right)$.

A sequence $\left(e_{0}, e_{1}, \ldots, e_{n}\right)$ of primitive idempotents in $A$ is called connected if $e_{i+1} N e_{i} \neq(0)$ for $i=0, \ldots, n-1$ and we denote the maximal length of connected sequence by $l(\Lambda)$.

Proposition 4. Let $\Lambda$ be semi-primary. Then gl.dim $\Lambda / N^{2}=l(\Lambda)=l\left(\Lambda / N^{3}\right)$ and $N^{l(\Lambda)+1}=(0)$.

Proof. It is clear that $l(\Lambda) \geq l\left(A / N^{2}\right)$. If $e N f \neq(0)$ and $e N f \subseteq N^{2}$, then we may assume $e N f \subseteq N^{s}, e N f \nsubseteq N^{s+1}$. Then $e N f=e N^{s} f$. Hence, there exist primitive idempotents $e_{0}=f, e_{1}, \ldots, e_{s}=e$ such that $e_{i+1} N e_{i} \nsubseteq N^{2}$. Hence, $\left(f, e_{1}, \ldots\right.$, $\left.e_{s-1}, e\right)$ is a connected sequence in $A$ and $\Lambda / N^{2}$. Therefore, $l(\Lambda) \leq l\left(\Lambda / N^{2}\right)$. We know gl.dim $\Lambda / N^{2}=l\left(\Lambda / N^{2}\right)$ by [11], Proposition 2 .

THEOREM 5. Let $A$ be a semi-primary ring with radical $N$. Then the following conditions are equivalent.

1) $A$ is a g.t.a. matrix ring over semi-simple rings.

2) $l(\Lambda)<\infty$.

3) gl.dim $A / N^{2}<\infty$.

4) $A$ is a homomorphic image of an hereditary semi-primary ring $\Omega$ such that $l(\Lambda)=l(\Omega)$. (cf. [2], Theorem 4.1 and [11], Theorem 5).

Proof. 1) $\rightarrow 2$ ). Let $\Lambda=T_{n}\left(R_{i} ; M_{i, j}\right) ; R_{i}$ semi-simple rings and $\Gamma=T_{n}\left(R_{i} ; 0\right)$. Then $A=\Gamma \oplus N$. Since we can replace idempotents in a connected sequence by isomorphic ones, we may assume that idempotents in a sequence are in $\Gamma$. Then $e_{i+1} N e_{i} \neq(0)$ implies $\rho(i)<\rho(i+1)$, where $e_{i} \in R_{\rho}(i)$. Hence, every length of connected sequence does not exceed $n$.

$2) \rightarrow 3$ ). It is clear from Proposition 4 .

$3) \rightarrow 4)$. Let $E_{1}, \ldots, E_{n}$ be mutually orthogonal idempotents in $A$ such that $E_{i} \Lambda E_{i} / E_{i} N E_{i}$ is a simple component of $\Lambda / N$. Since $l(\Lambda)<\infty, E_{i} N E_{i}=(0)$. Let $\Gamma=\sum \oplus E_{i} \Lambda E_{i} \subset A$. We use a similar argument to [11], $\S 2$. Put $Q=\Gamma \oplus$

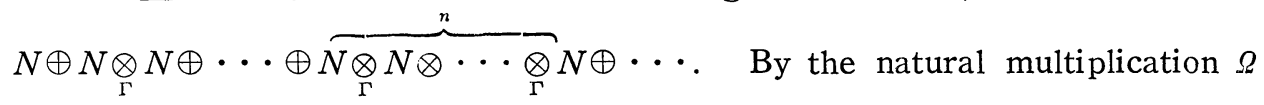
becomes a ring. If $\overbrace{N \otimes N \cdot \cdots \otimes}^{s} N \neq(0)$, then there exist idempotents $e_{i}, f_{i}$ in 
$\Gamma$ and $n_{i}$ in $N$ such that $e_{s} n_{s} f_{s} \otimes \cdots \otimes e_{1} n_{1} f_{1} \neq 0$. Hence, $e_{i} N f_{i} \neq(0)$ and $f_{i+1} e_{i}$ $\neq 0$ for all $i$, which means $f_{i+1} \approx e_{i}$ and $f_{i+1} N f_{i+1} \neq(0)$. Therefore, $N \overbrace{\otimes \cdots \otimes}^{l(\Lambda)+1}$ $=(0)$. It is clear that $N(\Omega)=N \oplus N \otimes N \oplus \cdots=\Omega \underset{\Gamma}{\otimes}$. We have a natural epimorphism $\varphi$ of $\Omega$ to $\alpha$ by setting $\varphi\left(r+n_{1}+n_{2} \otimes n_{3}+\cdots\right)=r+n_{1}+n_{2} n_{3}$ $+\cdots)$. From the construction of $\Omega$ we know that $\Omega$ is hereditary and $l(\Omega)$ $\geq l(1)$. However $N(2)^{l(\Lambda)+1}=(0)$. Hence, $l(\Omega) \leq l(\Lambda)$ by the following proposition 5 .

4) $\rightarrow 1$ ). It is clear from Theorem $4^{\prime \prime \prime}$.

Remark 5. From Proposition 4 and Theorems 2 and 5 we obtain $l(\Lambda)=$ gl.dim $\Lambda / N^{2} \geq$ gl.dim $\Lambda / \mathfrak{L}$ for any twoside ideal $\mathfrak{A}$ of $\Lambda$.

\section{Applications.}

In this section we shall give some properties of hereditary semi-primary rings as applications of results in $\S \S 1-3$.

From Theorems 4, 4" and 4"' we have

THeORem 6. Let $A$ be an hereditary semi-primary ring with nilpotency $n$ of the radical. Then for any two-sided ideal $\mathfrak{U}$ in $\Lambda$

$$
\text { gl.dim } \Lambda / \mathfrak{R} \leq n-1 \text {. }
$$

Furthermore, if $\Lambda / N$ is a directsum of $m$ simple rings and $N+\mathfrak{H} / N$ is a directsum of s simple rings, then

$$
\text { gl.dim } \Lambda / \mathfrak{A} \leq m-s+1 .
$$

(cf. [4], Theorem 8 and [11], Proposition 7).

The following proposition shows that the first inequality in the theorem is best, which was given in [4], Corollary 11.

$\mathrm{P}_{\mathrm{ROPOSITION}}$ 5. Let $\Lambda$ be an hereditary semi-primary ring. Then $l(\Lambda)=l\left(\Lambda / N^{2}\right)$ $=\operatorname{gl} \operatorname{dim} \Lambda / N^{2}$ is equal to (the nilpotency of $\left.N\right)-1$.

Proof. From Theorem 4'" we know $l(A)+1=$ the nilpotency of $N$.

Proposition 6. Let $A$ be an hereditary semi-primary ring. If $A / N$ is a simple ring, then so is $\Lambda$.

PROPOSITION 7. The center of an hereditary semi-primary ring $A$ is a directsum of fields. Especially $A$ is indecomposable if and only if its center is a field. 
Proof. We may assume that $A$ is isomorphic to a g.t.a. matrix ring $T_{n}$ ( $R_{i}: M_{i, j}$ ) over simple rings $R_{i}$. It is clear that each component of center $C$ of $A$ is contained in the center of $R_{i}$. We denote the center of $R_{i}$ by $C_{i}$. For $M_{i, j} \neq(0)$ we put $L_{i, j}=\left\{c \mid \in C_{i}\right.$, there exists a unique element $c^{\prime}(c)$ in $C_{j}$ such that $c m=m c^{\prime}(c)$ for all $\left.m \in M_{i, j}\right\}$, and $R_{j, i}=\left\{c^{\prime} \mid \in C_{j}\right.$, there exists a unique element $c\left(c^{\prime}\right)$ in $C_{i}$ such that $m c^{\prime}=c\left(c^{\prime}\right) m$ for all $\left.m \in M_{i, j}\right\}$. It is clear that $L_{i, j}$ and $R_{j, i}$ are fields and are isomorphic each other. We consider a path from the index 1 to $i ; i_{1}=1, i_{2}, \ldots, i_{r}=i$ such that $M_{i_{k}, i_{k+1}} \neq(0)$ or $M_{i_{k+1}, i_{k}} \neq$ (0) for all $k$. By $I$ we denote all indexes which is connected to 1 by the above path. Then $A=\Lambda_{I} \oplus \Lambda_{I^{c}}$ where $\Lambda_{I}$ consists of all elements in $\Lambda$ whose $(i, j)$. components are zero for $i, j \in I^{c}$. If $\Lambda$ is indecomposable, then every index is connected to 1 . Therefore, in this case we know from the above observation that $C$ is isomorphic to a subfield of $\bigcap_{i} R_{1} k_{i}$. Hence in general $C$ is a directsum of fields.

Proposition 8. Let $\Lambda$ be an hereditary semi-primary ring. We assume $\Lambda$ is indecomposable and $K$ is the center of $\Lambda . \quad \Lambda \underset{K}{\otimes} L$ is hereditary and semi-primary for all extension field $L$ of $K$ if and only if $\Lambda / N$ is separable over $K$, ( $K$-dim $A / N$ $=0$ ).

Proof. By Theorem $4^{\prime \prime} A$ is isomorphic to a g.t.a. matrix ring $T_{n}\left(R_{i} ; M_{i, j}\right)$ over simple rings $R_{i}$. If $\Lambda \underset{K}{\otimes} L$ is hereditary, then $R_{i} \underset{K}{\otimes} L$ is hereditary by Lemma 2 , since $\Lambda \otimes_{K} L=T_{n}\left(R_{i} \otimes_{K} L ; M_{i, j} \otimes_{K} L\right)$. Since $\Lambda \otimes_{K} L$ is semi-primary, so is $R_{i} \underset{K}{\otimes} L$. Let $C_{i}$ be the center of $R_{i}$. Then $C_{i} \underset{K}{\otimes} L$ is the center of $R_{i} \underset{K}{\otimes} L$. Hence $C_{i} \otimes_{K} L$ is a directsum of fields by Proposition 7. Therefore, $R_{i} \underset{K}{\otimes} L$ is a semi-simple ring with minimum conditions for any $L$ by $[10]$, p. 114, Theorem 1. Thus, we obtain from [6], Theorem 1 that $\left[R_{i}: K\right]<\infty$. Since $C_{i}$ is separable over $K$, so is $R_{i}$. Conversely, if $A / N$ is separable over $K$, then $[A /$ $N: K]<\infty$ by [13], Theorem 1. Hence, $N \underset{K}{\otimes} L$ is the radical of $\Lambda \underset{K}{\otimes} L$. Since $N$ is $\Lambda$-projective, $N \underset{K}{\otimes} L$ is $\Lambda \otimes L$-projective. Therefore, $\Lambda \underset{K}{\otimes} L$ is semi-primary and hereditary.

Lemma 10. Let $\Lambda$ be a g.t.a. matrix ring $T_{n}\left(\Delta_{k} ; M_{i, j}\right)$ and $\Gamma=T_{n}\left(\Delta_{1}, \ldots\right.$, $0, \ldots, \Delta_{n} ; M_{k, j}=(0)$ if $k$ or $\left.j=i\right)$. If $\Lambda$ is hereditary then so is $\Gamma$.

Proof. Let $e=T_{n}\left(0, \ldots, i_{i}, 0 \ldots ; 0\right)$ and $E=1-e$. Then $\Gamma=E \Lambda E . \quad E A$ 
$=E \Lambda E \oplus E \Lambda e . \quad E A e$ is a left ideal of $\Gamma^{\prime}=T_{n-i+1}\left(\Delta_{i}, \ldots, \Delta_{n} ; M_{k, j}\right)$. Hence, $E A e$ is $\Gamma^{\prime}$-projective. Furthermore, by Lemma 3 we obtain $0=1 \cdot \operatorname{dim}_{\Gamma} E A e=$ $1 . \operatorname{dim}_{\Gamma}{ }^{\prime} E \Lambda e=1 . \operatorname{dim}_{\Gamma} E$.1e, where $\Gamma^{\prime \prime}=T_{n-i}\left(\Delta_{i+1}, \ldots, \Delta_{n} ; M_{k, j}\right)$. Therefore, $E \Lambda$ is $\Gamma$-projective. Since $N(\Gamma)=E N E, E N E$ is $\Gamma$-projective.

TheOREM 7. Let $\Lambda$ be an hereditary semi-primary ring and $M$ a finitely generated projective A-module. Then $\operatorname{Hom}_{\Lambda}(M, M)$ is hereditary and semi-primary and so is eAe for any idempotent element $e$ in $A$.

Proof. $M \approx \sum_{j=1}^{k} \oplus\left(\Lambda e_{i(j)}\right)^{s_{j}}$. Hence, $\operatorname{Hom}_{\Lambda}(M, M)=T_{k}\left(e_{i(j)} \Lambda e_{i(j)}\right) s_{j} ; e_{i(k)} \Lambda e_{i(p)}$ $\left.\left(s_{k} \times s_{p}\right)\right)$, which is an induced g.t.a. matrix ring from $\Gamma=T_{k}\left(e_{i(j)} A e_{i(j)} ; e_{i(p)} A e_{i(q)}\right)$. From Lemma 10 we know $\Gamma$ is hereditary and semi-primary. Hence, so is $A$ and $e \Lambda e=\operatorname{Hom}_{\Lambda}(\Lambda e, \Lambda e)$ is hereditary.

Proposition 9. Let $\Lambda$ be an hereditary semi-primary ring and $M$ a projective left $A$-module. Then the annihilator ideal $\mathfrak{H}$ of $M$ in $A$ is a direct summand of $A$ as a left module and $\Lambda / \mathfrak{U}$ is hereditary.

Proof. We may assume that $\Lambda / N$ is a directsum of division rings. Since $M \approx \sum_{i=1}^{t}\left(\Lambda e_{i}\right)^{s_{i}}, \mathfrak{A}$ is equal to the annihilator ideal of $\sum_{i=1}^{t} \Lambda e_{i}$. If we denote the annihilator ideal of $\Lambda e_{i}$ in $A$ by $\mathfrak{H}_{i}$, then $\mathfrak{A}=\bigcap_{i} \mathfrak{A}_{i}$. Let $\Lambda=T_{n}\left(\Delta_{i} ; M_{i, j}\right)$ and $e_{i}=T_{n}\left(0,1_{\rho(i)}, 0 ; 0\right)$. Then $\Lambda e_{i}=T_{n}\left(0, \ldots, A_{\rho}(i), 0 ; M_{k, l}^{\prime}=(0)\right.$ for $l \neq \rho(i)$, $\left.M_{k, \mathrm{p}(i)}^{\prime}=M_{k, \mathrm{p}(i)}\right)$. It is clear that $\mathfrak{H}_{i} e_{k}=\Lambda e_{k}$ if $M_{k, \mathrm{p}(i)}=(0)$ and $\mathfrak{A}_{i} e_{k}=(0)$ if $M_{k, p(i)} \neq(0)$. Hence, $\mathfrak{U}_{i}=\sum \lambda e_{l^{\prime}}$. Therefore, $\mathfrak{U}=\sum_{s=1}^{p} \Lambda e_{l(s)}$. We note that $M_{l(p), k}$ $=(0)$ if $k \neq$ some $l(q)$. Hence, $\Lambda / \mathfrak{A}=e \Lambda e$ for some idempotent element $e$. Therefore, $\Lambda / \mathfrak{A}$ is hereditary from Theorem 7 .

Proposition 10. Let $\Lambda$ be as above. Then there exists a minimal faithful left ideal $L$ and every faithful left $\Lambda$-projective module contains an isomorphic image of $L$ as a direct summand.

Proof. Let $\Lambda / N \approx \sum \oplus \Delta_{i}$ and $T_{n}\left(R_{i} ; \mathfrak{M}_{i, j}\right)$ a g.t.a. matrix ring as a normal right representation, namely $R_{i}=\sum_{k=\rho(i)}^{p(t+1)-1} \oplus \Delta_{k}, M_{p, q}$ is a $\Delta_{p}-\Delta_{q}$ module and $\mathfrak{M}_{i, j}$ is as in (8) and furthermore, each row of $\mathfrak{M}_{i, j}$ is non-zero. From this assumption we can see as above that $A E_{1}=L$ is a faithful left ideal in $\Lambda$ where $E_{1}=T_{n}\left(1_{1}, 0 ; 0\right)$. Let $M$ be a faithful projective $\Lambda$-module. Since $M \approx \sum \oplus$ $\left(\Lambda e_{i}\right)^{s_{i}}$ and $e_{t} M \neq(0)$ for primitive idempotent $e_{t}$ in $\Lambda E_{1}, M \approx \Lambda E_{1} \oplus M^{\prime}$. 
Corollary 5. Every hereditary semi-primary ring is a subdirectsum of finite many of hereditary rings in the endomorphism ring of vector space over a division ring.

Proof. Let $L$ be a minimal faithful left ideal and $L=\Lambda e_{1} \oplus \cdots \oplus \Lambda e_{t}$. Then it is clear that $\Lambda e_{i}(i=1, \ldots, t)$ is a right module over a simple ring ( $\Delta_{i}$-module in the above). Hence, we have the corollary from Proposition 9.

As a related problem to Corollary 5 , we consider hereditary rings in the endomorphism ring of finitely generated module $M$ over a division ring $\Delta$. Let $\Lambda$ be such a ring and $N=N(\Lambda)$. Then we have a chain of $\Lambda$-module : $M \supset$ $N M \supset \cdots \supset N^{t-1} M \supset N^{t} M=(0)$. We put $\tilde{\Lambda}=\left\{x \mid \in \operatorname{Hom}_{\lrcorner}(M, M), x N^{i} M \subseteq N^{i} M\right.$ for all $i$. $\quad M=M_{1} \oplus M_{2} \oplus \cdots \oplus M_{t}$ as a $\Delta$-module such that $N^{i} M=M_{i+1} \oplus \cdots$ $\oplus M_{t}$. Then it is clear that $\tilde{\Lambda}=T_{t}\left(\Delta_{n_{1}}, \ldots, \Delta_{n_{t}} ; \Delta\left(n_{i} \times n_{j}\right)\right)$ where $\left[M_{i}: \Delta\right]$ $=n_{i}$, and $N(\tilde{\Lambda})^{t}=(0), N(\tilde{\Lambda})^{t-1} \neq(0)$.

LeMmA 11. $\Delta(p \times q) \underset{\Delta q}{\otimes}(q \times s) \approx \Delta(p \times s)$.

Proof. Let $e_{i}=i\left(\begin{array}{c}0 \\ 1,0 \cdot 0 \\ 0\end{array}\right)$ in $\Delta(p \times q)$. Then $\Delta(p \times q)=\Sigma \oplus e_{i} \Delta_{q}$. Hence $\Delta(p \times q) \underset{\Delta q}{\otimes} \Delta(q \times s)=\sum e_{i} \otimes \Delta(q \times s)$. It is clear that $e_{i} \otimes \Delta(q \times s) \approx i\left(\begin{array}{c}0 \\ \Delta \cdot \cdots \Delta \\ 0\end{array}\right)$.

From this lemma and Corollary 2 we know that $\tilde{A}$ is hereditary.

Propositron 11. Let $\Lambda$ be an hereditary semi-primary ring in a simple ring $\Delta_{n}$ with nilpotency $t$ of $N$ and we assume that $A$ has the unit element of $\Delta_{n}$. Then there exists a maximal hereditary semi-primary ring in $\Delta_{n}$ with nilpotency $t$ which contains $\Lambda$.

Proof. Let $\tilde{\Lambda}$ be as above, $T$ be an hereditary semi-primary ring containing $\tilde{\Lambda}$ and let its radical $N^{\prime}$ have the nilpotency $t$. We may assume $\Gamma=\widetilde{T}$. We consider a chain; $M \supset N^{\prime} M \supset \cdots \supset N^{\prime t-1} M \supset(0)$. It is clear that this chain is a composition series as a $\Gamma$-module. Furthermore, this is a chain as a $\tilde{\Lambda}$ module and $M$ has a composition series of length $t$ as a $\tilde{A}$-module. Hence, $N^{\prime} M \supseteq N M, N^{\prime 2} M \supseteq N N^{\prime} M \supseteq N^{2} M \cdots$. Hence $N^{i} M=N^{\prime^{i}} M$. Therefore, $\widetilde{\Gamma}=\tilde{\Lambda}$.

Proposition 12. Let $\Delta_{n}$ be a simple algebra over a field $K$ and $\Lambda$ an hereditary algebra with $[\Lambda: K]<\infty$. We assume that $\Lambda$ contains the unit element of $\Delta_{n}$ and its radical has the nilpotency $t$. $\Lambda$ is a maximal hereditary ring with nilpotency 
$t$ in $\Delta_{n}$ if and only if $\Lambda / N \approx \sum_{i=1}^{t} \oplus \Delta_{n_{i}}$ and $\sum_{i=1}^{t} n_{i}=n$.

Proof. The "only if" part is clear from Proposition 11. We prove the "if" part. From the assumption and Theorem $4^{\prime \prime \prime} \Lambda=T_{t}\left(R_{i} ; M_{i, j}\right) ; T_{i}$ is a simple algebra and $M_{i, j} \neq(0)$. Let $1=e_{1}+e_{2}+\cdots+e_{l}$, where $e_{i}$ is the unit element in $R_{i}$. Further $e_{i}=\sum_{j=1}^{n(i)} e_{i, j}$ is a decomposition of $e_{i}$ into the mutual orthogonal primitive idempotents in $\Delta_{n}$. Then we may assume that those $e_{i, j}^{\prime} s$ are a subset of matrix units in $\Delta_{n} . \quad e_{i} \Lambda e_{i}\left(\approx \Delta_{n_{i}}\right) \subseteq e_{i} \Delta_{n} e_{i}=\Delta_{n(i)}$. Hence, $n_{i} \leq n(i)$. However since $\sum n_{i}=n, n_{i}=n(i)$ for all $i$. Therefore, $e_{i} \Delta e_{i}=e_{i} \Delta e_{i}$ since $[\Lambda: K]<\infty$. Furthermore, $e_{i} \Delta_{n} e_{j}=e_{i} \Delta_{n} e_{i} M_{i, j} e_{j} \Delta_{n} e_{j}=M_{i, j}$, since $M_{i, j} \neq(0)$.

\section{Hereditary rings with minimum conditions}

In this section we shall study hereditary rings with left or right minimum conditions. Such a ring is also semi-primary. Hence, all results in $\S \S 2$ and 3 are valid for this ring. However, we give another approach to those results.

First we consider $A$ such that $\Lambda / N=\sum \oplus \Delta_{i}$. Let $1=\sum e_{i}$ as in $\S 3$. For any idempotent element $e$ we define $l(e)$ as follows: $l(e)=$ the composition length of $\lambda e$ as a left ideal. We can arrange $\left\{e_{i}\right\}$ as $l\left(e_{i}\right) \geq l\left(e_{i+1}\right)$ for all $i$. Then we have similar results for $l\left(e_{i}\right)$ to $n\left(e_{i}\right)$. From Lemma 5 , we have Lemma 7 for $l\left(e_{i}\right)$ and etc.

Lemma 12. Let $A$ be a g.t.a. matrix ring $T_{n}\left(\Delta_{i} ; M_{i, j}\right)$ over division rings $\Delta_{i}$. Then $l\left(e_{i}\right)=\sum_{j=i+1}^{n}\left[M_{j, i}: \Delta_{j}\right]$, where $e_{i}=T_{n}\left(0, \ldots, 1_{i}, 0, \ldots ; 0\right)$.

Thus, we have from Theorem $4^{\prime \prime \prime}$

THEOREM 8. $A$ is an hereditary ring with left minimum condition if and only if $\Lambda$ is isomorphic to a g.t.a. matrix ring $T_{n}\left(R_{i} ; M_{i, j}\right)$ over semi-simple rings $R_{i}$, which satisfies the conditions in Theorem $4^{\prime \prime}$ and $M_{i, j}$ is a finitely generated $R_{i}$-module for all $i>j$.

We note we do not have a relation as Lemma 8 for $l\left(e_{i}\right)$ and that there are no relations between $l\left(e_{i}\right)$ and $n\left(e_{i}\right)$ in general.

Proposition 13. Let $\Lambda$ be an hereditary g.t.a. matrix ring $T_{n}\left(\Delta_{i} ; M_{i, j}\right)$ over division rings. Let $t_{k}=\left[\bar{M}_{j, k}: \Delta_{j}\right]$. Then $l\left(e_{i}\right)=1+\sum_{k>i} t_{k} l\left(e_{k}\right)$, where $\bar{M}_{j, k}=M_{j, k} /$ $\sum_{l} M_{j, l} M_{l, k}$.

Proof. 


$$
N e_{i} / N^{2} e_{i}=\left(\begin{array}{l}
\frac{0}{\bar{M}_{i+1, i}} \\
\cdot \\
\cdot \\
\bar{M}_{n, 1}
\end{array}\right)
$$

and $\bar{M}_{j, i}$ is a left $\Delta_{j}$-module. Hence, since $N e_{i}$ is $\Lambda$-projective, $N e_{i}=\sum \oplus\left(\Lambda e_{k}\right)^{t_{k}}$ and $N e_{i}$ is a maximal $\Lambda_{i}$-module in $\Lambda e_{i}, l\left(e_{i}\right)=\sum t_{k} l\left(e_{k}\right)+1$.

Proposition 14. Let $A$ be an hereditary semi-primary ring such that $\Lambda / N=$ $\sum_{i=1}^{n} \oplus \Delta_{i}$. Then $N^{n}=(0)$. Furthermore, $N^{n-1} \neq(0)$ if and only if $e_{i+1} \Lambda e_{i} \neq(0)$ for all $i$. In this case 1) $e_{i} \Lambda e_{j} \neq(0)$ for all $i \geq j$. 2) $\left[e_{i} \Lambda e_{j}: \Delta_{i}\right] \geq\left[e_{i} \Lambda e_{j},: \Delta_{i}\right],\left[e_{i} \Lambda e_{j}:\right.$ $\left.\Delta_{j}\right] \geq\left[e_{i} \Lambda e_{j},: \Delta_{j},\right] \quad\left(\operatorname{resp} .\left[e_{i} \Lambda e_{j}: \Delta_{j}\right] \geq\left[e_{i^{\prime}} \Lambda e_{j}: \Delta_{j}\right],\left[e_{i} \Lambda e_{j}: \Delta_{i}\right] \geq\left[e_{i}, \Lambda e_{j}: \Delta_{i^{\prime}}\right]\right)$ if $j^{\prime} \geq j\left(\right.$ resp. $\left.i \geq i^{\prime}\right)$. 3) $l\left(e_{i}\right)>l\left(e_{i+1}\right)$. 4) If A satisfies the left minimum condition, then $l(e)>l\left(e^{\prime}\right)$ is equivalent to $n(e)>n\left(e^{\prime}\right)$.

Proof. We put $M_{i, j}=e_{i} \Lambda e_{j}$. Then $\Lambda$ is a g.t.a. matrix ring $T_{n}\left(\Delta_{i} ; M_{i, j}\right)$. Hence $N^{n}=(0)$. $\quad N^{n-1}=M_{n, n-1} M_{n-1, n-2} \cdots M_{2,1}$. Since $\varphi_{i, k}^{j}$ is monomorphic by Theorem $1, N^{n-1} \neq(0)$ if and only if $e_{i+1} \Lambda e_{i}=M_{i+1, i} \neq(0)$ for all $i$. We assume that all $M_{i+1, i} \neq(0)$. Then $\left.M_{i, j} \supseteq M_{i, i-1} M_{i-1, i-2} \cdot \cdot M_{j+1, j} \neq(0) .2\right)$ If $j^{\prime} \geq j$, then $M_{i, j} \supseteq M_{i, j} M_{j^{\prime}, j}$. Hence $\left[M_{i, j}: \Delta_{i}\right] \geq\left[M_{i, j},: \Delta_{i}\right]$ and $\left[M_{i, j}: \Delta_{j}\right] \geq$ $\left[M_{i, j^{\prime}}: \Delta_{j}\right]$ since $\varphi$ is monomorphic. 3) By Lemma 12 and 2) we obtain $l\left(e_{i}\right)$ $\left.=\sum_{j=i+1}^{n}\left[M_{j, i}: \Delta_{j}\right]>\sum_{j=i+2}^{n}\left[M_{j, i+1}: \Delta_{j}\right]=l\left(e_{i+1}\right) .4\right)$ is clear from definition of $l()$ and $n($ ).

Proposition 15. Let $\Lambda$ be as in Proposition 13. If $N^{n-1}$ is a non-zero irreducible left $\Lambda$-module, then $\Delta_{i}$ is monomorphic to $\Delta_{i+1}$ and $\Lambda$ satisfies the left minimum condition.

Proof. $N^{n-1}=M_{n, n-1} \cdots M_{2,1}$. Since $\Lambda e_{n}=e_{n} \Lambda e_{n}=\Delta_{n}, N^{n-1}$ is an irreducible $\Delta_{n}$-module and hence, $\left[N^{n-1}: \Delta_{n}\right]=1$. Furthermore, $N^{n-1}$ contains an isomorphic image of $M_{n, n-1} \cdots M_{i+1, i}$ as a left 1 -module. Hence $\left[M_{i+1, i}: \Delta_{i+1}\right]=1$, say $M_{i+1, i}=\Delta_{i+1} m_{i+1}$. Since $M_{i+1, i}$ is a right $\Delta_{i}$-module, $\delta m_{i+1}=m_{i+1} \delta^{\prime}$ for some $\delta^{\prime} \in \Delta_{i}$. It is clear that a mapping $\delta \rightarrow \delta^{\prime}$ is a monomorphism of $\Delta_{i+1}$ to $\Delta_{i}$.

LeMmA 13. Let $\Lambda$ be an hereditary g.t.a. matrix ring over $\Delta_{i}$ such that $\left[M_{i, j}\right.$ : $\left.\Delta_{i}\right]=\left[M_{i, j}: \Delta_{j}\right]=1$ for all $i, j$. Then $\Lambda$ is isomorphic to a usual tri-angular matrix ring over $\Delta$, where $\Delta \approx \Delta_{i}$. 
Proof. First we choose a generator $m_{i, i-1}$ of $M_{i, i-1}$ for $i=2, \ldots, n$; $M_{i, i-1}=m_{i, i-1} \Delta_{i-1}$. We define a generator $m_{i, j}$ of $M_{i j}$ as follows: $m_{i, j}=m_{i, i-1}$ $\cdots m_{\jmath+1, j}$. Since $\Lambda$ is hereditary, $m_{i, j} \neq 0$. It is clear from the definition of $m_{i, j}, m_{i, j} m_{j, k}=m_{i, k}$. As in the proof of Proposition 15 we obtain an isomorphism $\alpha_{i, j}$ of $\Delta_{i}$ to $\Delta_{j}$ such that $x m_{i, j}=m_{i, j} x^{\alpha_{i, j}}$ for $x \in \Delta_{i}$. Since $m_{i, j} m_{j, k}=m_{i, k}$, we have $\alpha_{i, j} \alpha_{j, k}=\alpha_{i, k}$. Let $\Gamma$ be a usual tri-angular matrix ring $T_{n}(\Delta ; \Delta)$ over 4. We define a mapping $\Phi$ of $\Gamma$ to $A$ as follows: for an element $\gamma=T\left(\gamma_{i, j}\right)$ $\Phi(\gamma)=T_{n}\left(\gamma_{i, j}^{\alpha_{n, i}} m_{i, j}\right)$, where $m_{i, i}=1_{i}$. Then we can easily check, by noting the above observation, that $\Phi$ is isomorphic.

Theorem 9. Let $\Lambda$ be an hereditary ring such that $\Lambda / N$ is a directsum of $n$ division rings. Then the following conditions are equivalent:

1) $\Lambda$ is a usual tri-angular matrix ring over a division ring.

2) $A$ is a general uniserial ring and $N^{n-1} \neq(0)$.

3) $\left[e_{n} \Lambda e_{1}: e_{1} \Lambda e_{1}\right]=\left[e_{n} \Lambda e_{1}: e_{n} \Lambda e_{n}\right]=1$ and $N^{n-1} \neq(0)$.

4) $l\left(e_{1}\right), r\left(e_{n}\right) \leq n$, and $N^{n-1} \neq(0)$.

5) $n\left(e_{1}\right)=l\left(e_{1}\right)=r\left(e_{n}\right)$ and $N^{n-1} \neq(0)$.

Where $N$ is the radical of $\Lambda$ and $\left\{e_{i}\right\}$ is the set of non-isomorphic primitive idempotent elements as the beginning of this section, and $r(e)$ is the composition length of $\mathrm{e} \Lambda$ as a right $\Lambda$-module.

Proof. From the assumption and Theorem 4", we know that $\Lambda=T_{n}\left(\Delta_{i}\right.$; $M_{i, j}$ ), where the $\Delta_{i}$ 's are division rings.

1) $\rightarrow 2$ ) is clear.

2) $\rightarrow 3$ ). Since $\Lambda e_{i}$ has a unique composition series, we obtain that $\left[M_{i, j}\right.$ : $\left.\Delta_{i}\right]=1$ (resp. $\left.\left[M_{i, j}: \Delta_{j}\right]=1\right)$ for all $i \geq j$.

$3) \rightarrow 4)$. By Proposition 14 we have $\left[M_{i, j}: \Delta_{i}\right] \leq\left[M_{n, 1}: \Delta_{n}\right]=1$. Hence $l\left(e_{1}\right), r\left(e_{n}\right) \leq n$.

4) $\rightarrow 5)$. 4) implies clearly that $l\left(e_{i}\right)=n-i+1$ for all $i$ and hence $\left[M_{i, j}\right.$ : $\left.\Delta_{i}\right]=1$. Therefore, $n\left(e_{i}\right)=l\left(e_{i}\right)$.

5) $\rightarrow 1$ ). Since $n\left(e_{i}\right)=n\left(e_{i+1}\right)+1$ by Lemma $8, n\left(e_{1}\right), r\left(e_{n}\right)=n$. Therefore, $\left[M_{i, j}: \Delta_{i}\right]=\left[M_{i, j}: \Delta_{j}\right]=1$ as above. Hence, we have 1) from Lemma 12.

Remark 7. In Theorem 9 if we replace the assumption " $A / N$ is a directsum of $n$ division rings" by simple rings, then Theorem 9 is true provided we replace 1) by $\left.1^{\prime}\right): \Lambda$ is a g.t.a. matrix ring $T_{n}\left((\Delta)_{s_{i}} ; M_{i, j}=\left(s_{i} \times s_{j}\right)\right.$-matrices over $\left.\Delta\right)$. 


\section{Appendix.}

Let $R$ be a discrete rank one valuation ring with quotient field $K$ and $\Sigma$ $=K_{n}$. We know, by [8], Theorem 6.2, all types of hereditary orders over $R$ in $\sum$. We shall give another proof by a similar argument to $\S 4$.

By standard argument (cf. [8], §1) we may assume that $R$ is complete. In this case we can use Lemma 5. Let $p$ be a unique maximal ideal in $R$ and $\left\{e_{i}\right\}$ the set of primitive idempotent elements as in $\S 1$. Then $e l e$ is an hereditary order in $e \sum e$, where $e=\sum_{i} e_{i}$ and $e \Lambda e / e N e$ is a directsum of division rings.

First, we assume that $\Lambda=e \Lambda e$ and $\Sigma=e \sum e$. Since $\sum e_{i}$ is an irreducible left ideal in $\sum$ by [1], Proposition 2.8, we may assume that $e_{i}$ is a matrix unit $e_{i, j}$ in $\sum$. Furthermore, we may assume that $\Lambda$ is contained in a maximal order $\Omega=R_{n}$. By [8], Lemma 3.2 we know that $N(\Omega)=\mathfrak{p} \Omega \leq N$. Let $\vec{A}=\Lambda$ / $N(\Omega)$.

Lemma A. If $N e_{i} \approx \Lambda e_{j}$, then 1) $e_{j, i} \in \Lambda, N e_{i}=\Lambda e_{j, i}, N e_{i} / p \Omega e_{i}$ is $\Lambda / p$ p-projective and $l\left(\bar{e}_{i}\right)=1+l\left(\bar{e}_{j}\right)$ or 2$) N e_{i}=\Lambda \mathrm{p} e_{j, i}$.

Proof. Let $\varphi: \Lambda e_{j} \rightarrow N e_{i}$ be an isomorphism. $\varphi\left(e_{j}\right)=n e_{i}=e_{j} n e_{i}$ for some $n \in N$. Since $p \in N$, there exists $\lambda$ in $\Lambda$ such that $\lambda e_{j} n e_{i}=p e_{i}=e_{i} \lambda e_{j} n e_{i}$. Hence, $p=e_{i} \lambda e_{j} n e_{i} . \quad$ Therefore, 1) $e_{i} \lambda e_{j}=p \varepsilon e_{i, j}$ and $e_{j} n e_{i}=\varepsilon^{\prime} e_{j, i}$ or 2) $e_{i} \lambda e_{j}=\varepsilon$, and $e_{j} n e_{i}$ $=p \varepsilon^{\prime} e_{j, i}$, where $\varepsilon, \varepsilon^{\prime}$ are unit elements in $R$. Case 1). $N e_{i}=\Lambda e_{j} n e_{i}=\Lambda e_{j i}$ and $\varphi\left(\mathfrak{Q} \Omega e_{j}\right)=\mathfrak{p} \Omega e_{j, i}=\mathfrak{p} \Omega e_{i} . \quad$ Hence, $N e_{i} / \mathfrak{p} \Omega e_{i} \approx \Lambda / \mathfrak{p} \Omega \vec{e}_{j} . \quad$ Since $\bar{\Lambda} \bar{e}_{i, i}$ is irreducible, $l\left(\bar{e}_{i}\right)=l\left(\bar{e}_{j}\right)+1$.

LEMmA B. $\Lambda / p \Omega$ is an hereditary ring with minimum conditions.

Proof. Since $N=\sum N e_{i}, N / p \Omega=\sum \oplus N / p \Omega \bar{e}_{i}$ is $\Lambda / p \Omega$-projective by Lemma $\mathrm{A}$ and a fact that $N e_{i}$ is indecomposable.

Lemma C. We assume $l\left(\bar{e}_{i}\right) \geq l\left(\bar{e}_{i+1}\right)$ for all $i$, then $l\left(\bar{e}_{i}\right)>l\left(\vec{e}_{i+1}\right)$.

Proof. We assume that $l\left(\bar{e}_{n}\right)<l\left(\bar{e}_{n-1}\right)<\cdots<l\left(\bar{e}_{i+1}\right)$. Since for $n<j \leq i+1$ $N e_{j} \approx \Lambda e_{j+1}$ by Lemma A. Hence $e_{j+1} N e_{j} \approx e_{j+1} \Lambda e_{j+1}$. Therefore, $\bar{e} \bar{\Lambda} \bar{e}=T_{n-i}(R /$ $\left.\mathfrak{p} ; M_{i, j}=R / \mathfrak{p}\right)$ by Theorem $4^{\prime}$ and the assumption $\Omega \supseteq \Lambda$, where $e=\sum_{k=1+1}^{n} e_{k}$. If $l\left(\bar{e}_{i+1}\right)=l\left(\bar{e}_{i}\right)$, then $N e_{i} \approx \Lambda e_{i+2}$ by Lemma A. Hence, $\overline{e^{\prime}} \bar{\Lambda} \overline{e^{\prime}}=T_{n-i+1}\left(R / \mathfrak{p} ; M_{i+1, i}\right.$ $=(0), M_{k, l}=R / p$ for $\left.(k, l) \neq(i+1, i)\right)$, where $e^{\prime}=e_{i}+e$. Therefore, $N$ has the same components on $i^{\text {th }}$ and $i+1^{\text {th }}$ columns. Since $\Lambda=\operatorname{Hom}_{\Lambda}(N, N)$ by [7], 
Theorem 6.1, $A \ni e_{i+1, i}, e_{i, i+1}$, which is a contradiction.

From this lemma we have that $e_{i} \Lambda e_{j}=R$ for $i \geq j$. Therefore, we have

Theorem. Let $R$ be a discret rank one valuation ring with quotient field $K$. An hereditary order over $R$ in $K_{n}$ is isomorphic to

$$
\left|\begin{array}{ccc}
R & \mathfrak{p} & \mathfrak{p} \\
\cdots & \cdots & \cdots \\
R & R & \mathfrak{p} \\
\cdots & \cdots & \cdots \\
R & R & R \\
\cdots & \cdots & \cdots
\end{array}\right|
$$

where $p$ is a unique maximal ideal in $R$.

\section{REFERENCES}

[1] M. Auslander and O. Goldman, Maximal order Trans. Amer. Math. Soc. 97 (1960), $1-24$.

[2] S. U. Chase, A generalization of the ring of triangular matrices, Nagoya Math. J. 18 (1961), 13-25.

[3] S. Eilenberg, Homological dimension and syzgies, Ann Math. 64 (1956), 328-336.

[4] S. Eilenberg, H. Nagao and T. Nakayama, On the dimension of modules and algebras, IV, Nagoya Math. J. 10 (1956), 87-96.

[5] M. Harada, Note on the dimension of modules and algebras J. Inst. Polyt. Osaka City Univ. 7 (1956), 17-27.

[6] M. Harada, On homological theorems of algebras, ibid, 10 (1959), 123-127.

[ 7 ] M. Harada, Multiplicative ideal theory in hereditary orders, J. Math. Osaka City Univ. 14 (1963), 83-106.

[ 8 ] M. Harada, Structure of hereditary orders over local rings, ibid, 14 (1963), 1-22.

[ 9 ] M. Harada, Hereditary orders, Trans. Amer. Math. Soc. 107 (1963), 273-290.

[10] N. Jacobson, Structure of Rings, Amer. Math. Soc. Press. (1956).

[11] J. P. Jans and T. Nakayama, On the dimension of modules and algebras, VII, Nagoya Math. J. 11 (1957), 67-76.

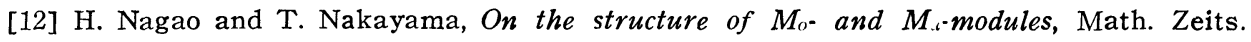
59 (1953), 164-170.

[13] A. Rosenberg and D. Zelinsky, Cohomology of infinite algebras, Trans. Amer. Math. Soc. 82 (1956), 85-98.

[14] T. Nakano, A nearly semi-simple ring, Comm. Math. Univ. Sancti Pauli 7 (1959), 27-33.

Osaka City University 